\title{
Preparation of nano-structured cryptomelane materials for catalytic oxidation reactions
}

\author{
S. Said ${ }^{1} \cdot$ M. $\operatorname{Riad}^{1} \cdot$ M. Helmy ${ }^{1} \cdot$ S. Mikhail $^{1} \cdot$ L. Khalil $^{2}$
}

Received: 24 February 2016/Accepted: 31 March 2016/Published online: 4 May 2016

(C) The Author(s) 2016. This article is published with open access at Springerlink.com

\begin{abstract}
Manganese-based octahedral molecular sieves of the type K-OMS-2 (cryptomelane structure) were prepared via reflux method by synproportionation of $\mathrm{KMnO}_{4}$ and $\mathrm{Mn}^{2+}$ in acidic aqueous suspension. For this method, different manganese anions (sulphate, chloride and acetate) were used, which exert a strong influence on the prepared materials. Several techniques such as X-ray diffraction, Fourier transformer infrared, Raman spectroscopy, transmission electron microscopy, differential and gravimetric thermal analysis and $\mathrm{H}_{2}$-temperature programmed reduction were used to characterize the prepared samples. The results revealed that the prepared samples were mainly pure mono-phase cryptomelane materials. The obtained K-OMS-2 material on using the sulphate anion has more available lattice oxygen as compared to that prepared by the other anions. The catalytic activity of the prepared samples was tested towards the oxidative dehydrogenation of cyclohexane. Over the K-OMS-2RS sample, cyclohexane conversion was significantly higher than the other prepared samples.
\end{abstract}

Keywords Octahedral molecular sieves - Manganese oxide Cyclohexane $\cdot$ Catalytic oxidation reaction . Cyclohexene

M. Riad

maryriad2006@yahoo.com

1 Egyptian Petroleum Research Institute, Cairo, Egypt

2 Faculty of Science, Ain Shames University, St. Cairo, Egypt

\section{Introduction}

Catalytic partial oxidation of hydrocarbons "particularly alkanes", using oxygen or air as oxidant, is significant and economical for the chemical industry. Among various alkane oxidations, the selective oxidation of cyclohexane is much attractive because of its formed product, cyclohexene.

Cyclohexene is used as an intermediate for the production of cyclohexanol, which is dehydrogenated to give cyclohexanone, a precursor to caprolactam. Caprolactam is the precursor to Nylon 6 , a widely used synthetic polymer.

Cyclohexene is also a precursor to adipic acid, maleic acid, dicyclohexyl adipate, and cyclohexene oxide and can be used as a specific solvent. This reaction is operated in liquid phase using heterogeneous catalysts, but required high temperature $\left(\sim 150^{\circ} \mathrm{C}\right)$, besides high selectivity $(>80 \%)$ to the sum of cyclohexanol and cyclohexanone only could be observed at low cyclohexane conversion [1]. Modi and Trivedi, [2] studied the catalytic behaviour of zeolite-Y-entrapped $\mathrm{Ru}(\mathrm{III})$ and $\mathrm{Fe}(\mathrm{III})$ complexes over the oxidation of cyclohexane forming cyclohexanone and cyclohexanol. Zeolite-Y-entrapped Ru showed higher catalytic activity $(41.1 \%)$ with cyclohexanone (84.6\%) selectivity. Therefore, the development of effective homogeneous catalysts could offer advantages.

Cryptomelane (K-OMS-2), an octahedral molecular sieve, is an allotropic form of manganese oxide having a well-defined tunnel structure. K-OMS-2 constructed from edge-shared double chains $\mathrm{MnO}_{6}$ octahedra units that are corner connected to form an open tunnel of 4.6 by $4.6 \mathrm{~A}^{\circ}$ size. Potassium ions are situated inside the tunnels to provide charge balance and to stabilize the structure (chemical composition $=\mathrm{KMn}_{8} \mathrm{O}_{16} \cdot \mathrm{nH}_{2} \mathrm{O}$ ). The diameters of the tunnel cross sections are in the range of that for 
typical zeolite pores. Due to its porous structure, hydrophobic nature, easy release of lattice oxygen, acidic sites and mixed valence of manganese species, low costefficient and environmentally friendly, cryptomelanes have been extensively explored for potential applications such as molecular sieves which are used as catalyst. They have gained a good performance in redox catalysis alternative to the conventional catalyst such as supported noble metals [3]. Several different morphologies have been synthesized, ranging from micrometer-long fibres to nanorods and paper-like materials [4].

Different methods have been reported for preparing K-OMS-2 such as: reflux [5] basic [6], reduction in the presence of surfactant [7] and sonication [8]. From these methods, a variety of cryptomelane materials were obtained with different morphology, crystallite size, evolution of lattice oxygen and enhanced activity in various oxidation processes.

Schurz et al. [9] prepared K-OMS-2 via the reflux method and studied the influence of cationic precursors $\mathrm{KMnO}_{4}$ and $\mathrm{Ba}(\mathrm{MnO} 4)_{2}$ on the synthesis product and tested for the oxidation of benzyl alcohol with molecular oxygen in liquid toluene at $110{ }^{\circ} \mathrm{C}$. The catalytic activity of the K-OMS-2 is higher than Ba-OMS-2 materials, which directly correlated to their higher surface area.

Sithambarama et al. [10] prepared K-OMS-2 by the reflux method and investigated their activity for hydrogen generation via the water-gas shift reaction.

Sun et al. [11] prepared K-OMS-2 materials by solidstate and reflux methods to study the catalytic oxidation of toluene. The results established that K-OMS-2 prepared by the refluxing method exhibited higher catalytic activity than that by the solid-state reaction method. It was apparent that the high catalytic activity of the prepared K-OMS-2 prepared by the refluxing method attributed to the more available lattice oxygen and the higher oxygen mobility.

Deng et al. [12] prepared K-OMS-2 with different rodlike, fibrous-like and nest-like morphologies by solid-state reaction, reflux and hydrothermal methods, respectively. The materials were tested for liquid-phase oxidation of $p$ chlorotoluene to $p$-chlorobenzaldehyde. Over nest-like $\mathrm{K}-\mathrm{OMS}-2, p$-chlorotoluene conversion was significantly higher than that of the others.

Hndel et al. [13] prepared cryptomelane successfully at ambient pressure and temperature by a simple one-step reaction pathway based on redox reactions of either $\mathrm{MnSO}_{4}$ or $\mathrm{MnCl}_{2}$ together with $\mathrm{KMnO}_{4}$ in aqueous solution.

Almquist et al. [14] prepared cryptomelane via three methods, reflux, milling and sol gel, and tested for ethanol oxidation. The results reveal that the sample prepared via the reflux method has the highest surface area compared to the other prepared samples and higher ethanol conversion.
Duan et al. [15] prepared (OMS-2) via reflux method and tested for the photodegradation of Acid Orange 7 in aqueous solutions; the catalyst shows enhanced activity and stability within the reaction time.

Accordingly, the common method for preparing K-OMS-2 is the reflux method, which has many advantages over the prepared cryptomelane materials such as redox property, oxygen vacancies, oxygen mobility and high surface area. These advantages resulted in the enhanced catalytic activity in different reaction processing.

In the current work, different K-OMS-2 materials were prepared via the reflux method. The diversity of the properties of the obtained materials depending on the type of anionic precursors including morphologies, crystallite size and lattice oxygen were investigated. The catalytic activity of the prepared samples towards oxidation reaction of cyclohexane was also recorded using flow system operated under atmospheric pressure.

\section{Experimental}

\section{Preparation of K-OMS-2}

K-OMS-2 was prepared through the oxidation of manganese sulphate by potassium permanganate in an acidic medium under reflux according to the procedure described by DeGuzman et al., [16]. A solution of $0.4 \mathrm{M}$ potassium permanganate $(13.3 \mathrm{~g})$ was dissolved in $225 \mathrm{ml}$ of distilled water at room temperature, and $6.7 \mathrm{ml}$ of nitric acid ( $65 \mathrm{wt} \%$ ) was added to adjust the $\mathrm{pH}$ value to " 3.5 ". This solution was added to a second one consisting of $19.8 \mathrm{~g}$ $\mathrm{MnSO}_{4}(1.75 \mathrm{M})$ dissolved in $67.5 \mathrm{ml}$ of distilled water (under continuous stirring). The resulting solution was transferred to a $500 \mathrm{ml}$ round-bottomed flask fitted with a reflux condenser, stirred vigorously and refluxed at a temperature of $100{ }^{\circ} \mathrm{C}$ for $24 \mathrm{~h}$. The formed dark brown precipitate is filtered, washed until $\mathrm{pH}=7$ and then dried at $120{ }^{\circ} \mathrm{C}$ overnight. $\mathrm{MnSO}_{4} \cdot \mathrm{H}_{2} \mathrm{O}$ was replaced with either $\mathrm{MnCl}_{2} \cdot 4 \mathrm{H}_{2} \mathrm{O}$ or $\mathrm{Mn}\left(\mathrm{CH}_{3} \mathrm{OO}\right)_{2} \cdot 4 \mathrm{H}_{2} \mathrm{O}$, to investigate the influence of the anions on the texture and the structure of the prepared K-OMS-2 materials. The procedure is completed as described for manganese sulphate, except that for manganese acetate, the nitric acid is replaced by acetic acid. The prepared samples are denoted as K-OMS-2RS, K-OMS-2RC and K-OMS-2RA, respectively.

\section{Structural phase changes}

Different techniques were applied to investigate the physico-chemical characteristics of the prepared K-OMS-2 materials. 


\section{$X$-ray powder diffraction analysis $(X R D)$}

The structure and phase purity of the prepared materials were analysed via X-ray diffraction (XRD). The experiments were carried out using a Shimadzu XD-1 diffractometer using $\mathrm{Cu} K_{\alpha}$ radiation $(\lambda=0.1542 \mathrm{~nm})$ at a beam voltage of $40 \mathrm{kV}$ and $40 \mathrm{~mA}$ beam current.

The intensity data were collected at room temperature in a $2 \theta$ range of $10^{\circ}-70^{\circ}$ with a scan rate of $0.7 \mathrm{~s}^{-1}$. The joint committee on Powder Diffraction Society (JCPDS) database was used to index the peaks of XRD.

Average crystallite size calculated from X-ray line broadening using Sherrer's equation at $2 \theta=37.5^{\circ}$ characterized the prepared materials.

\section{Fourier transformer infrared spectroscopy}

Fourier transformer infrared (FTIR) spectroscopy experiments were performed on spectrometer Perkin-ElmerSpectrum-1 in the range of 4000 to $400 \mathrm{~cm}^{-1}$ at a resolution of $4 \mathrm{~cm}^{-1}$. The samples were ground to a fine powder and dispersed in $\mathrm{KBr}$ to compress into pellets before measurements.

\section{Raman spectroscopy}

Raman spectra were recorded at room temperature in the range of $100-800 \mathrm{~cm}^{-1}$ with an HR-UV 800 confocal scanning spectrometer (Horiba Jobin Yvon), equipped with a Peltier-cooled charge-coupled device $(1152 \times 298$ pixels) with spectral resolution of $0.5 \mathrm{~cm}^{-1}$. The Raman scattering was excited using a $632.81 \mathrm{~nm}$ excitation wavelength supplied by an internal He-Ne laser through an Olympus high-stability BXFM confocal microscope.

\section{Thermal analysis}

Differential thermal (DTA) and thermo-gravimetric (TG) analyses were performed to study the structural changes of the prepared materials with thermal treatment. A $10 \mathrm{mg}$ of sample was heated up to $1100{ }^{\circ} \mathrm{C}$, with a heating rate of $10{ }^{\circ} \mathrm{C} \mathrm{min}{ }^{-1}$ in an airflow at a rate of $50 \mathrm{ml} \mathrm{min}^{-1}$ on SDTQ-600 (TA-USA) thermo balance instrument.

\section{High-resolution transmission electron microscope (HRTEM)}

The morphologies of the prepared materials were studied using high-resolution transmission electron microscope (HRTEM). The images of the materials were obtained in JEOL JEM-2100F microscope operating at voltage of $200 \mathrm{kV}$. The samples were prepared by dispersing in ethanol and sonicated for $20 \mathrm{~min}$. The suspension dropped on a carbon-coated copper grid for TEM observations.

\section{Temperature-programmed reduction (TPR)}

Temperature-programmed reduction experiments were carried out to investigate the redox properties (the ease of reducibility of metal oxide) for the studied materials. The experiments performed in automatic equipment (Chembet 3000, Quantachrome). Typically, $100 \mathrm{mg}$ of calcined material was loaded into a quartz reactor and heated under inert atmosphere $\left(20 \mathrm{ml} / \mathrm{min} \mathrm{N}_{2}\right)$ at $200{ }^{\circ} \mathrm{C}$ for $3 \mathrm{~h}$ prior to running TPR experiments, and cooled down to room temperature in $\mathrm{N}_{2}$. Then the sample was submitted to a constant rate of heat treatment $\left(10{ }^{\circ} \mathrm{C} / \mathrm{min}\right.$ up to $\left.1000^{\circ} \mathrm{C}\right)$ in a gas flow (mixture $\mathrm{H}_{2} / \mathrm{N}_{2}, 5 / 95 \mathrm{vol} \%$, reducing gas) $80 \mathrm{ml} /$ min. A thermal conductivity detector (TCD) employed to monitor the amount of hydrogen consumption.

\section{Textural analysis}

The textural properties were determined from the $\mathrm{N}_{2}$ adsorption-desorption isotherms at liquid nitrogen temperature $\left(-196^{\circ} \mathrm{C}\right)$ and carried out using NOVA $3200 \mathrm{~S}$ Unite, automated gas sorption analyser (Quantachrome Corporation) system to investigate the textural properties of the prepared cryptomelane samples. All samples were degassed at $300{ }^{\circ} \mathrm{C}$ for $4 \mathrm{~h}$ in nitrogen atmosphere prior to adsorption to ensure a dry clean surface. The pore size distributions were determined from Barrett-Joyner-Halenda $(\mathrm{BJH})$ method applied to the desorption branch of the isotherm.

\section{Catalytic activity}

The oxidation of cyclohexane was carried out in a fixed bed vertical downward flow glass reactor (internal diameter $6 \mathrm{~mm}$ ), operated under atmospheric pressure. About $2 \mathrm{ml}$ of the pretreated catalyst was placed at the hot zone of the reactor, supported on either side with a thin layer of quartz wool and ceramic beads. The reaction temperature was monitored by a thermocouple placed in the middle of the catalyst bed. The catalyst was activated at $250{ }^{\circ} \mathrm{C}$ for $2 \mathrm{~h}$, before reaction in a controlled stream of air as a carrier gas

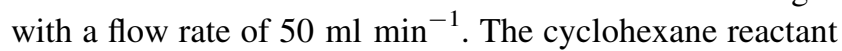
was fed into the reactor with the required flow rate $(3 \mathrm{ml} / \mathrm{h})$ using a syringe infusion pump. The collected products in the receiver flask were analysed in a gas chromatograph (Perkin-Elmer) equipped with a HP-1 capillary column $(60 \mathrm{~m} \times 0.32 \mathrm{~mm} \times 0.5 \mu \mathrm{m})$ and a flame ionization detector. 


\section{Results and discussions}

\section{X-ray diffraction analysis (XRD)}

X-ray diffraction patterns for the prepared K-OMS-2 material via the reflux method (using the different precursors) depicted in Fig. 1.

The pattern for the three different precursors reveals the appearance of diffraction peaks at $2 \theta=12.8^{\circ}, 18.5^{\circ}$, $28.9^{\circ}, 37.5^{\circ}, 42^{\circ}$ and $50^{\circ}$; these peaks are consistent with those of the standard cryptomelane (K-OMS-2) material with tetragonal symmetry corresponding to $\mathrm{KMn}_{8} \mathrm{O}_{16}$, (JCPDS 29-1020), which emphasized that the resulting material is a cryptomelane structure.

The peak intensities are the apparent difference between the three studied precursors, which reflect a difference in the formed crystallite sizes. It seems to increase form sulphate to chloride and then drastically increases for acetate.

XRD for K-OMS-2RS exhibits two additional diffraction peaks at $2 \theta=21.53^{\circ}$ and $46.03^{\circ}$ (marked with asterisk in Fig. 1), with low intensity. Most likely, these peaks related to the more difficult paths for the elimination by calcination of sulphate compared to acetate and oxalate, indexed as $\mathrm{K}_{2} \mathrm{SO}_{4}$ [16].

The average crystallite sizes (Table 1) are in the order: K-OMS-2RC $\quad(26.6 \mathrm{~nm})=\mathrm{K}$-OMS-2RS $<$ K-OMS-2RA $(30.4 \mathrm{~nm})$, in parallel with the decrease in the size of the studied anions $\mathrm{Cl}^{-}<\mathrm{SO}_{4}^{-2}<\mathrm{CH}_{3} \mathrm{COO}^{-}$and the decrease in the polarity $\mathrm{SO}_{4}^{-2}>\mathrm{Cl}^{-}>\mathrm{CH}_{3} \mathrm{COO}^{-}$. Thus, the acetate group has the largest size and the lowest polarity, which allows the cryptomelane particles to grow in large crystallite. Whereas sulphate and chloride species are difficult to

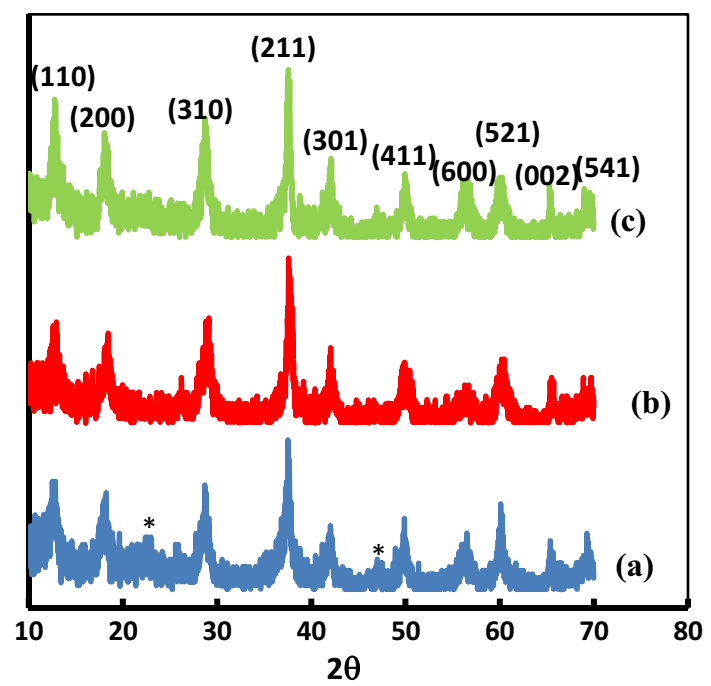

Fig. 1 The X-ray diffraction patterns of $a$ OMS-2RS, $b$ OMS-2RA, c OMS-2RC samples. ${ }^{*} \mathrm{~K}_{2} \mathrm{SO}_{4}$ eliminate compared to the acetate group and might be present as $\mathrm{K}_{2} \mathrm{SO}_{4}$ and $\mathrm{KClO}_{3}$ [16], which induce the dispersion of the formed crystallites and prevent their agglomeration.

\section{Fourier transformer infrared (FT-IR)}

The FT-IR spectra for the prepared samples depicted in Fig. 2Aa-c. The spectrum detects several bands at 462 , $516,682,1560$ and $3362 \mathrm{~cm}^{-1}$ (on using sulphate anion), which are shifted to $455,513,671,1584$ and $3369 \mathrm{~cm}^{-1}$ (on using chloride anion) and to 458, 517, 677, 1568 and $3360 \mathrm{~cm}^{-1}$ (on using acetate anion). These bands ascribed to the vibrations of the $\mathrm{MnO}_{6}$ octahedral framework, which present a clear signature of the cryptomelane structure [17].

The vibration band located at $\sim 462 \mathrm{~cm}^{-1}$, can be attributed to the vibration of the manganese species $\left(\mathrm{Mn}^{3+}\right)$ in the octahedral sites. The bands detected at $\sim 517$ and $682 \mathrm{~cm}^{-1}$ were assigned to the vibration due to displacement of the oxygen anions relative to the manganese ions along the direction of the octahedral chains, and the stretching mode of $\mathrm{MnO}_{6}$ octahedral along the double chain, respectively. The band appeared at $1120 \mathrm{~cm}^{-1}$ is attributed to Mn-O-H, structural vibrations [18].

Meanwhile, the broadband detected at $\sim 3369 \mathrm{~cm}^{-1}$ is assigned to the stretching vibration of the water molecule and $\mathrm{OH}^{-}$in the lattice of the cryptomelane material, and that at $\sim 1584 \mathrm{~cm}^{-1}$ is assigned to the bending vibration of water molecules in the tunnels and hydrogen-bounded $\mathrm{OH}-$ groups, which implies that the hydroxyl groups exist in the prepared material [19].

\section{Raman spectroscopy}

Raman spectroscopy for the prepared samples is depicted in Fig. 2Ba-c. The spectrum (K-OMS-2RS) reveals the appearance of Raman signals centred at 142, 250, 544 and $607 \mathrm{~cm}^{-1}$. The peak detected at $544 \mathrm{~cm}^{-1}$ is assigned to the vibration that is due to the displacement of the oxygen anion (relative to manganese ion) along the direction of the octahedral chains, whereas the vibration frequency at $607 \mathrm{~cm}^{-1}$ is assigned to $\mathrm{Mn}-\mathrm{O}$ stretching mode in tetrahedral sites environment, as confirmed by FT-IR. The signals at 142,250 and $360 \mathrm{~cm}^{-1}$ are due to the $\mathrm{Mn}-\mathrm{O}-\mathrm{Mn}$ bending vibration in the $\mathrm{MnO}_{2}$ octahedral lattice [20].

From $\mathrm{Mn}-\mathrm{O}$ stretching range, the two sharp, high-intensity, high-frequency Raman bands at 544 and $607 \mathrm{~cm}^{-1}$ are indicative of a well-developed tetragonal structure with an interstitial space consisting of $(2 \times 2)$ tunnels of cryptomelane material [21, 22].

For OMS-2RC and OMS-2RA prepared materials the Raman signals for cryptomelane materials are shifted from $142,167,350,569$ and 622 to $155,364,557$ and $611 \mathrm{~cm}^{-1}$, respectively, as shown in Fig. $2 \mathrm{Bb}$, c. 
Table 1 The texture properties and weight loss of the prepared K-OMS-2R samples

\begin{tabular}{|c|c|c|c|c|c|c|c|c|c|c|}
\hline \multirow[t]{3}{*}{ Sample name } & \multicolumn{5}{|c|}{ Textural parameters } & \multicolumn{5}{|c|}{ Thermal behaviour } \\
\hline & \multicolumn{2}{|c|}{$\begin{array}{l}\text { Crystallite } \\
\text { size (nm) }\end{array}$} & \multirow[t]{2}{*}{$\begin{array}{l}S_{\mathrm{BET}} \\
\left(\mathrm{m}^{2} / \mathrm{g}\right)\end{array}$} & \multirow[t]{2}{*}{$\begin{array}{l}T \cdot V_{p} \\
(\mathrm{cc} / \mathrm{g})\end{array}$} & \multirow[t]{2}{*}{$D_{p}(\mathrm{~nm})$} & \multicolumn{4}{|c|}{ Temperature ranges $\left({ }^{\circ} \mathrm{C}\right)$} & \multirow{2}{*}{$\begin{array}{l}\text { Total Weigh } \\
\text { loss }(\%) \\
25-1150\end{array}$} \\
\hline & XRD & TEM & & & & $25-250(\%)$ & $300-750(\%)$ & $750-950(\%)$ & $950-1150(\%)$ & \\
\hline K-OMS-2RS & 26.6 & 9 & 96 & 0.3 & 12 & 5 & 6.6 & 0.99 & 2.6 & 15.19 \\
\hline K-OMS-2RC & 26.6 & 8 & 68 & 0.2 & 3 & 4.93 & 5.42 & 3.05 & 3.08 & 16.48 \\
\hline K-OMS-2RA & 30.4 & 11 & 86 & 0.2 & 3 & 3.67 & 4.66 & 2.55 & 2.53 & 13.41 \\
\hline
\end{tabular}

Italic character is the total weight loss compared at different reaction temperature range

$S_{\mathrm{BET}}$ BET surface area, $V_{p}$ pore volume, $T \cdot V_{p}$ total pore volume, $D_{p}$ pore diameter

Fig. 2 A FT-IR, B Raman spectroscopy of $a$ OMS-2RS, $b$ OMS-2RA, $c$ OMS-2RC samples
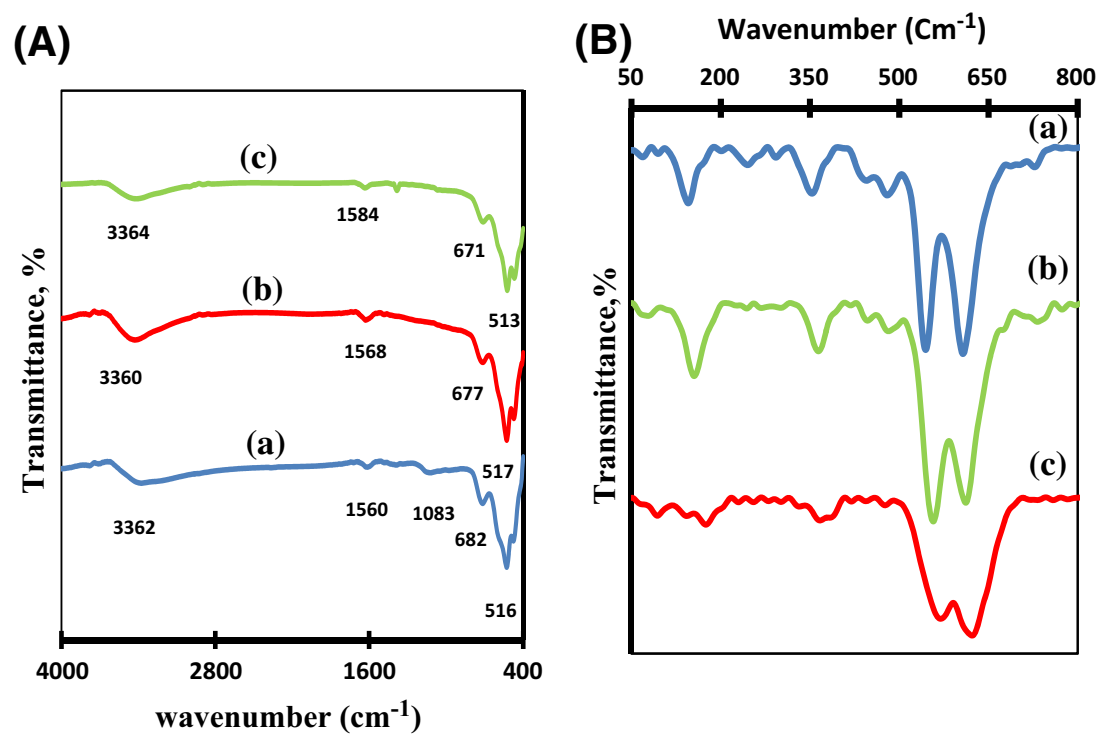

It is clear that the intensity of Raman bands at the range of $540-620 \mathrm{~cm}^{-1}$ increases from OMS-2RC to OMS-2RS and then OMS-2RA, which reveals a good tetragonal structure with an interstitial space consisting of $(2 \times 2)$ tunnels, developed for OMS-2RA compared with the other two samples.

In addition, the Raman signals are shifted to higher wave number in the order OMS-2RC $>$ OMS2RA $>$ OMS-2RS, which implies that the material prepared using manganese sulphate precursor contains higher amounts of oxygen vacancies.

\section{Differential and gravimetric thermal analysis (DTA and TGA)}

Differential and gravimetric thermal analysis profiles for the prepared samples are represented in Fig. 3A-C.

The DTA profile (Fig. 3Aa) of K-OMS-2RS sample displayed three endothermic peaks located at 569, 849 and $967{ }^{\circ} \mathrm{C}$ "centred at 555,855 and $969{ }^{\circ} \mathrm{C}$, as confirmed from the dDTA profile". The endothermic peak at $569{ }^{\circ} \mathrm{C}$ is an indication for desorption of water inside the $(2 \times 2)$ tunnels, as stated by Suib [3]. The second and third endothermic peaks correspond to the phase transformation of $\mathrm{MnO}_{2}$ into $\mathrm{Mn}_{2} \mathrm{O}_{3}$ and $\mathrm{Mn}_{2} \mathrm{O}_{3}$ into $\mathrm{Mn}_{3} \mathrm{O}_{4}$, respectively. The thermal behaviour for the K-OMS-2RC and K-OMS2RA samples is the same as for K-OMS-2RS one, but shifted to higher temperatures 633,879 and $1000{ }^{\circ} \mathrm{C}$ on using acetate, which implies its higher thermal stability.

These thermal effects were accompanied by steps of weight losses. The TG profile (Fig. 3Ab) demonstrates 5.0, 4.9 and $3.6 \%$ weight losses, for sulphate, chloride and acetate materials, respectively, at the temperature range $25-250{ }^{\circ} \mathrm{C}$, which could be due to the depletion of the physically and chemisorbed water molecules either on the surface and or on the channel structure. With the increase in the temperature to $750{ }^{\circ} \mathrm{C}$, the weight loss increases to 6.6, 5.4 and $4.66 \%$ (for K-OMS-2RS, K-OMS-2RC and $\mathrm{K}-\mathrm{OMS}-2 \mathrm{RA}$, respectively), which is due to the release of the water molecules inside the tunnels, besides the desorption of the structural oxygen close to the surface (related with the formation of lattice vacancies without 


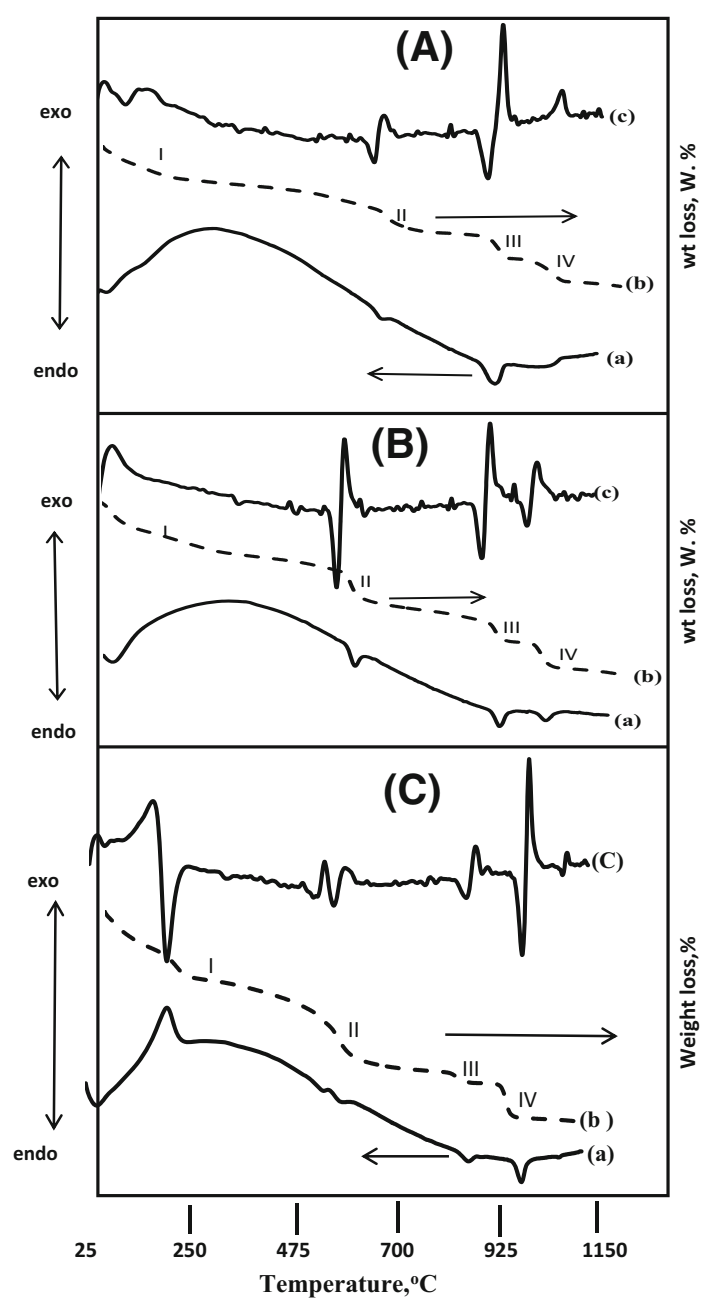

Fig. $3 a$ Differential thermal analysis (DTA), $b$ thermal gravimetric analysis (TGA), $c$ derivative differential thermal analysis (dDTA) of A K-OMS-2RS, B K-OMS-2RC, c K-OMS-2RA

decomposition of the material). A weight loss of $\sim 1.0,3.0$ and $2.3 \%$ at $750-950{ }^{\circ} \mathrm{C}$, and $2.6,3.1$ and $2.5 \%$ at up to $950{ }^{\circ} \mathrm{C}$ occurred on using K-OMS-2RS, K-OMS-2RC and K-OMS-2RA, respectively, which implies the transformation of cryptomelane to bixbyite $\left(\mathrm{Mn}_{2} \mathrm{O}_{3}\right)$ and then to hausmannite $\left(\mathrm{Mn}_{3} \mathrm{O}_{4}\right)$ species $[23,24]$, with total weight loss $15.20,16.40$ and $13.06 \%$. The total weight loss for acetate material (Fig. 3C) was found to be the lowest on "13.06" compared with the other ones, which indicates that K-OMS-2 material prepared on using the acetate anions was the most thermally stable one, in parallel with DTA behaviour.

Clearly, the weight losses at temperature range $250-750{ }^{\circ} \mathrm{C}$ are in the order sulphate $>$ chloride $>$ acetate. There are plenty of lattice oxygen in the produced OMS-2 material that prepared via the reflux technique on using manganese sulphate which become more easy to mobile upon thermal treatment (in agreement with the Raman spectroscopic results).

\section{Transmission electron microscopy (TEM)}

TEM was employed to investigate the morphologies of the prepared samples (Fig. $4 \mathrm{a}-\mathrm{c}$ ).

TEM image for nano-sized particles K-OMS-2RS material (Fig. 4a) shows agglomerated or clusters of disorderly stacked needle-like nanorods with an average diameter $9.0 \mathrm{~nm}$. The TEM image for K-OMS-2RC (Fig. 4b) exhibits fine needle-like nanorod morphologies, which have regular shape in which their ends are similar in diameter as their middle part with an average diameter of $8.0 \mathrm{~nm}$. Besides, the TEM image for K-OMS-2RA (Fig. 4c) shows dispersed needle-like crystallites, but with a larger average diameter of $11 \mathrm{~nm}$. It is evident that the degree of dispersion for the prepared cryptomelane particles on using either acetate and or sulphate is higher than that on using chloride, in agreement with Walanda et al.'s [25] study.

\section{$\mathrm{H}_{2}$-Temperature programmed reduction $\left(\mathrm{H}_{2}\right.$-TPR)}

$\mathrm{H}_{2}$-temperature programmed reduction for the prepared samples is depicted in Fig. 5a-c.

$\mathrm{H}_{2}$-TPR profile for OMS-2RS detects, two strong peaks at $383,403{ }^{\circ} \mathrm{C}$ and the others are broad weak at 450 , $650{ }^{\circ} \mathrm{C}$, according to the postulated studies. These peaks related to the successive reduction steps as follows:

$\mathrm{MnO}_{2} \rightarrow \mathrm{Mn}_{2} \mathrm{O}_{3}$,

$\mathrm{Mn}_{2} \mathrm{O}_{3} \rightarrow \mathrm{Mn}_{3} \mathrm{O}_{4}$,

$\mathrm{Mn}_{3} \mathrm{O}_{4} \rightarrow \mathrm{MnO}$,

$\mathrm{MnO} \rightarrow \mathrm{Mn}^{0}$.

As well known, the Mn-O bonds of K-OMS-2 are relatively weak and the Mn species in OMS-2 are reduced more easily at reduction temperature below $500{ }^{\circ} \mathrm{C}$. Besides, Liu et al. [26] stated that due to the bigger negative reduction potential of $\mathrm{MnO}$, no reduction band assignable to the $\mathrm{MnO} \rightarrow \mathrm{Mn}^{0}$ process was observed even up to $750{ }^{\circ} \mathrm{C}$. Therefore, the weak reduction peak observed at $650{ }^{\circ} \mathrm{C}$ related to the reduction of some of $\mathrm{K}_{2} \mathrm{SO}_{4}$, which still inter-chelated in the cryptomelane structure [27]. Therefore, $\mathrm{MnO}$ was the final state of cryptomelane- $\mathrm{MnO}_{2}$ reduction.

The TPR profile of K-OMS-2RC (Fig. 5b) is similar to that of sulphate, but the reduction peaks at 380 and $403{ }^{\circ} \mathrm{C}$ overlapped and appeared at $401{ }^{\circ} \mathrm{C}$. Two reduction peaks were observed at 403 and $453{ }^{\circ} \mathrm{C}$, the weak one at lower temperature is assigned to the reduction of $\mathrm{MnO}_{2} / \mathrm{Mn}_{2} \mathrm{O}_{3}$ to $\mathrm{Mn}_{3} \mathrm{O}_{4}$, and the strong one at higher temperature is related to the reduction of $\mathrm{Mn}_{3} \mathrm{O}_{4}$ to $\mathrm{MnO}$.

The $\mathrm{H}_{2-}$ TPR profile for the material prepared using manganese acetate as precursor (Fig. 5c) detected two 


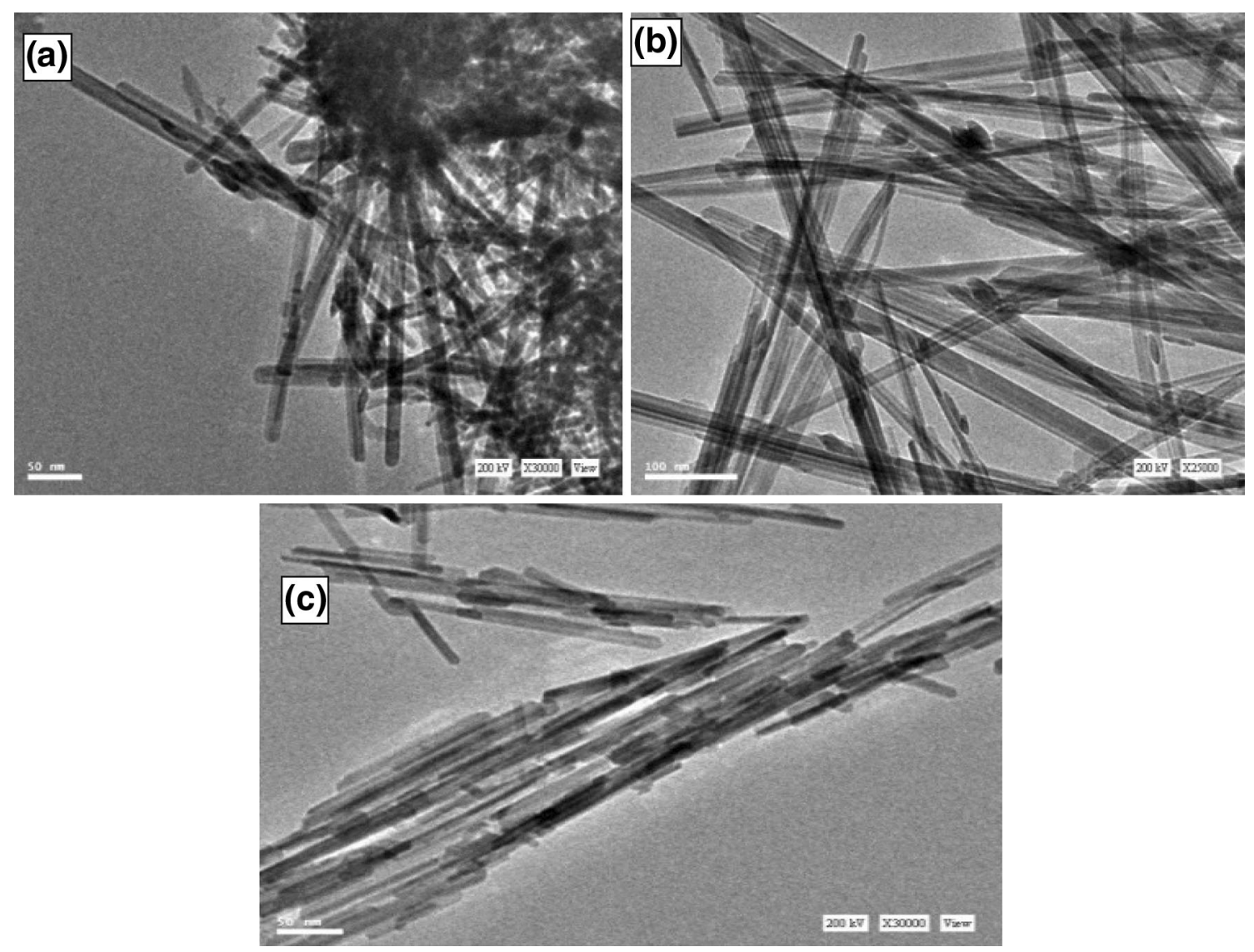

Fig. 4 TEM images of a OMS-2RS, b OMS-2RC, c OMS-2RA

reduction peaks at 349 and at $373{ }^{\circ} \mathrm{C}$. The profile is similar to that using manganese sulphate and chloride, but the reduction peak (high temperature one) shifted to lower temperature from 450 to $375{ }^{\circ} \mathrm{C}$ compared with the two other precursors. This implies that the reduction of cryptomelane seems to be easier, i.e. the reducibility and reactivity of manganese are higher. In other words, the $\mathrm{Mn}-\mathrm{O}$ bonds of OMS-2 on using manganese sulphate and manganese chloride precursors are stronger and reduced more difficultly than that for acetate.

\section{Textural properties}

The nitrogen adsorption-desorption isotherms (Fig. 6A) of the prepared K-OMS-2 needle-like nanorod samples show that the adsorption isotherms are related to type II according to Brunauer classification [28] and are characteristic for cryptomelane materials in accordance with Malinger et al. [29]. The isotherms of the samples exhibits H3-type hysteresis loop according to the IUPAC classification [30]. This type indicates the presence of the aggregation of plate-like nanoparticles giving rise to slitshaped pores, due to the interparticle voids of the aggregated K-OMS-2 nanorods, in agreement with the TEM images (Fig. 4). The isotherm for K-OMS-2RS and K-OMS-2RA samples exhibited a hysteresis loop in a broader relative pressure range of $0.75<P / P^{0}<1.0$, while the isotherm for K-OMS-2RC sample (Fig. 6A) reveals that the hysteresis loop nearly disappeared, suggesting the presence of capillary condensation without hysteresis taking place in the pores of the size range of wide micropores and/or narrow mesopores. Chloride anions can affect the morphology of the prepared OMS2RC via either expansion and or contraction of the interconnectivity between nearby pores causing the disappearance of the hysteresis. The BJH pore size distribution curve derived from the desorption branch (Fig. 6B) reveals the presence of bimodal mesopores for the K-OMS-2RS and K-OMS-2RA samples. However, the bimodal distribution is not obvious for the K-OMS-2RC sample. These results reflect the role of large anionic precursor (sulphate and acetate) in the creation of heterogeneous pore size distribution in comparing with the small size chloride species.

The texture properties of the prepared samples are listed in Table 1 . The surface area, total pore volume and average 


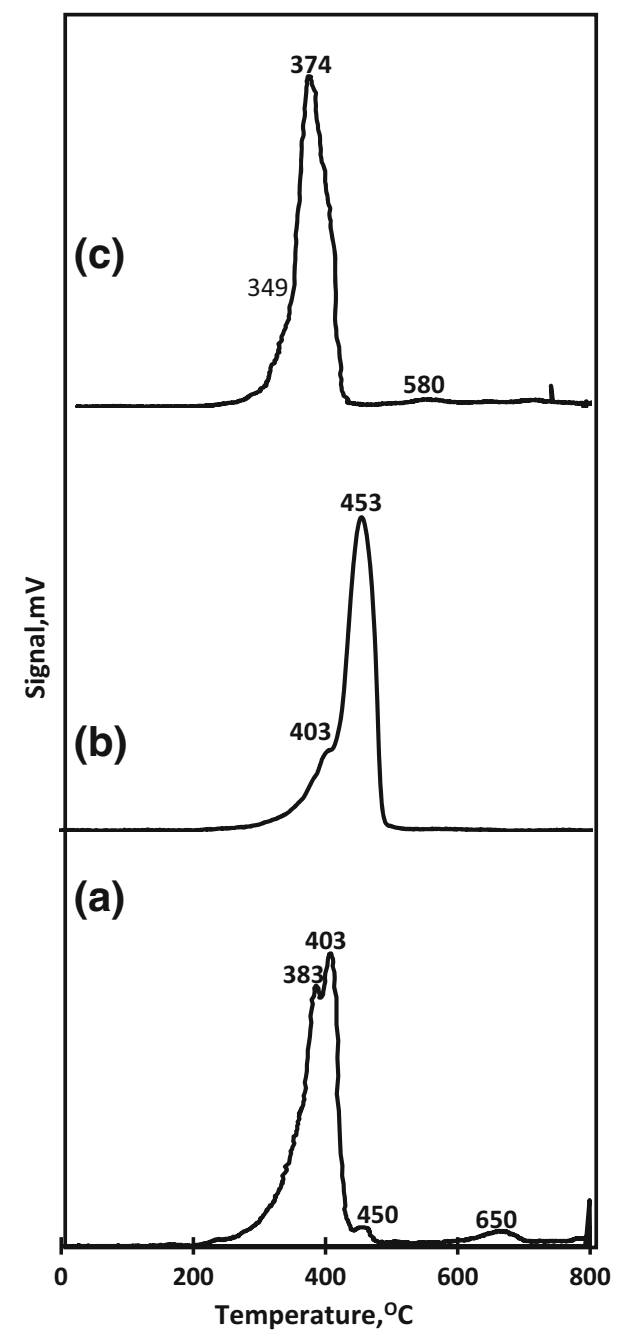

Fig. 5 TPR profiles of $a$ OMS-2RS, $b$ OMS-2RC and COMS-2RA samples

pore diameter of the K-OMS-2RS sample are $96 \mathrm{~m}^{2} \mathrm{~g}^{-1}$, $0.3 \mathrm{~cm}^{3} \mathrm{~g}^{-1}$ and $12 \mathrm{~nm}$ respectively, which are higher than those of the K-OMS-2RC and K-OMS-2RA samples. These results match the XRD data (Fig. 1) and TEM images (Fig. 4) in terms of the particle size. The agglomeration of the needle-like nanorods forming bunches shows mesopores between the needles; this structure is responsible for the high surface area and total pore volume exposed by the K-OMS-2RS sample.

However, the high surface area value and the average pore diameter are shown to have a high value of $12.0 \mathrm{~nm}$. This reflects the diffusion of the strong acidic sulphate species (large polar species) towards the narrow mesopores, allowing the opening up of some narrow regions to furnish a measurable area and expand the pores. Upon removing the sulphate species, it leaves behind pores with a relatively large diameter. Using the acetate group as precursor was able to increase the polarity of the K-OMS-
2, and the adsorption of non-polar molecular $\mathrm{N}_{2}$ hindered and decreased the measured BET area $\left(86 \mathrm{~g} / \mathrm{cm}^{2}\right)$.

The surface area for cryptomelane decreased on using chloride species as precursor compared with K-OMS-2RS, and becomes $68 \mathrm{~m}^{2} / \mathrm{g}$. This may be due to the accumulation of smaller particles of chloride species, which may still be contaminated with the formed cryptomelane material in the form of $\mathrm{KClO}_{3}$ on the pore wall and opening, leading to a shrinkage in pore structure, a decrease in total pore volume and a significant drop in the extent of surface area. Thus, the surface area can widely vary depending on the type of the anion precursors.

\section{Catalytic activity}

The catalytic oxidation of cyclohexane is performed in a flow-type system operating under atmospheric pressure and at the reaction temperature varying between 300 and $400{ }^{\circ} \mathrm{C}$, cyclohexane flow rate $3 \mathrm{ml} / \mathrm{h}$, liquid hourly space velocity $\left(\mathrm{LHSV}=1.5 \mathrm{~h}^{-1}\right.$ ) and with air flow rate of $50 \mathrm{ml} / \mathrm{min}$. The results are illustrated in Figs. 7, 8 and 9.

From data in Fig. 7, it is clear that the total conversions for all samples increased with the increase in reaction temperature up to $400{ }^{\circ} \mathrm{C}$. A low yield of liquid products obtained within the reaction temperature ranges $300-325^{\circ} \mathrm{C}$, and then sharply increases with further increase in the reaction temperature up to $400{ }^{\circ} \mathrm{C}$. The amount of gaseous products slowly increased with increasing temperature from 300 to $400{ }^{\circ} \mathrm{C}$.

The change in the activity of the prepared samples towards cyclohexane conversion is in the following sequence: K-OMS-2RS $>$ K-OMS-2RC $>$ K-OMS-2RA catalyst (histogram in Fig. 8).

From the liquid product distribution data obtained and given in Fig. 9, it is clear that the yield of the oxygenated products (cyclohexanon and cyclohexanal) increased with the increase in the reaction temperature. It exhibits low yield up to reaction temperature $350{ }^{\circ} \mathrm{C}$, then the yield sharply increases with further increase in temperature and reaches its maximum value $\sim 26 \%$ at $400{ }^{\circ} \mathrm{C}$ on both K-OMS-2RS and K-OMS-2RC. Meanwhile, the OMS2RA sample shows low yield towards the formation of oxygenated products.

On the other hand, the yield of the dehydrogenated products (cyclohexene) increased slightly with the gradual increase in the reaction temperature (for samples OMS2RA and OMS-2RC) and low yield of dehydrogenated products obtained on raising the reaction temperature to $400{ }^{\circ} \mathrm{C}$. For the sample OMS-2RS, the yield of dehydrogenated products show a maximum at the reaction temperature $350{ }^{\circ} \mathrm{C}(\sim 46 \%)$ and with a higher yield compared to the other two samples; then it decreases along with the increase in reaction temperature. The yield of 


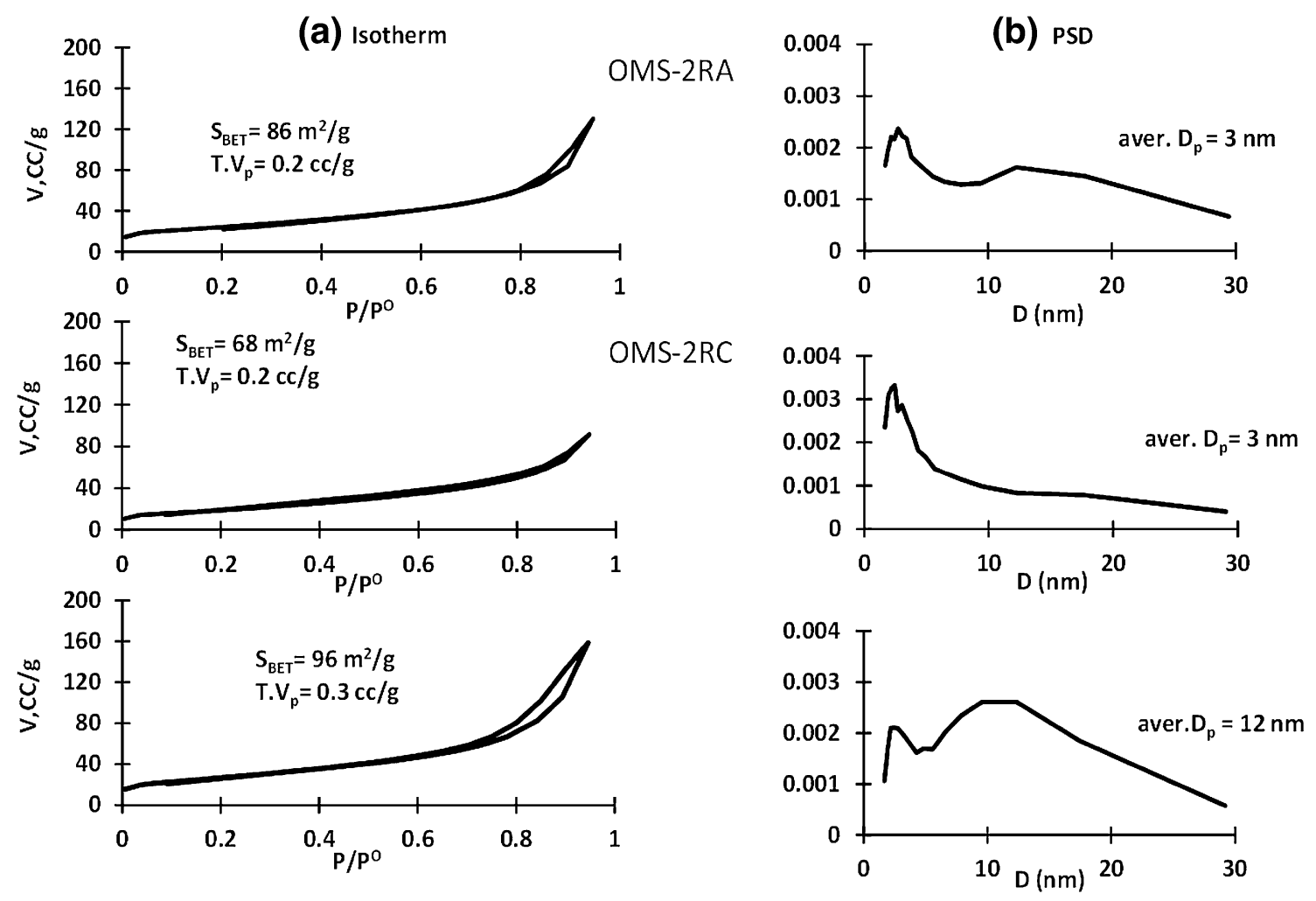

Fig. 6 a Nitrogen adsorption/desorption isotherms and $\mathbf{b}$ pore size distribution curves of K-OMS-2 samples

Fig. 7 Effect of reaction temperature on cyclohexane conversion over the K-OMS-2R samples
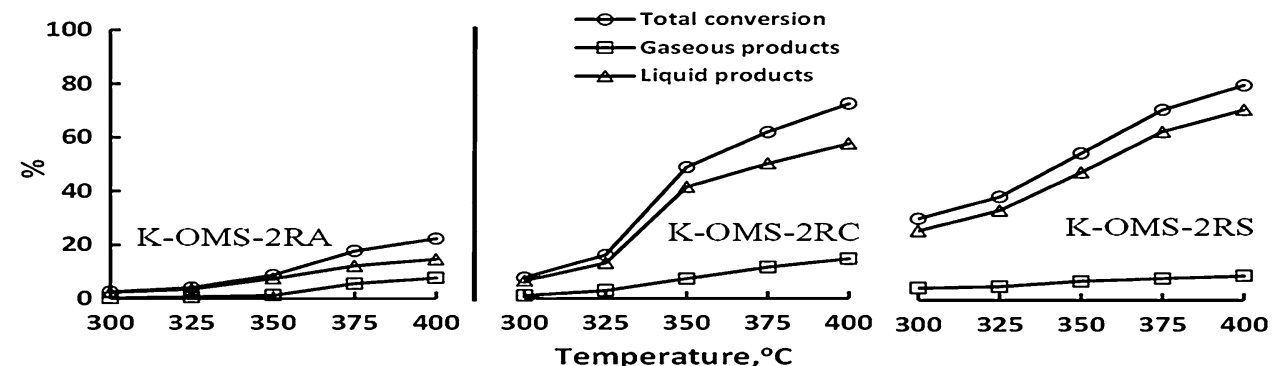

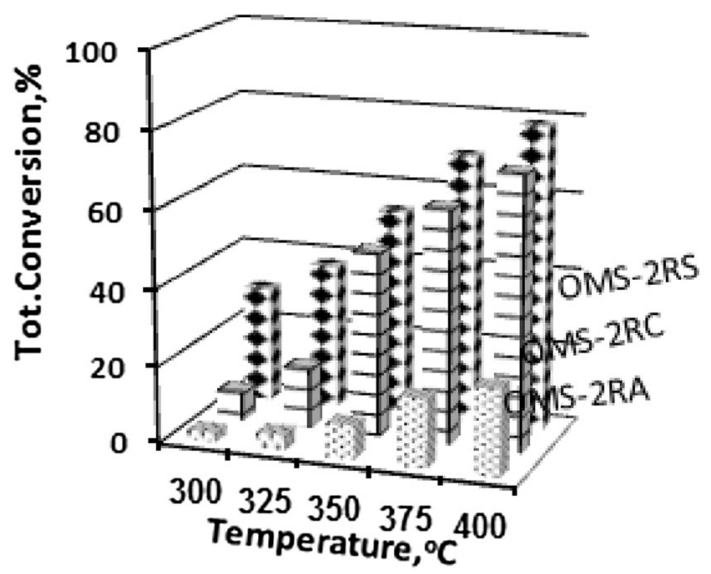

Fig. 8 The relation between the total conversion and the reaction temperature over the K-OMS-2R samples alkylated and oxygenated products continue their increase with the gradual increase in reaction temperature, also with low yield for sample OMS-2RA (Fig. 9A).

Concurrently, the selectivity of the three prepared samples (OMS-2RA, OMS-2RS and OMS-2RC) towards the formation of the dehydrogenated products (cyclohexene) decreased with the increase in reaction temperature, which is parallel with the increase in the selectivity towards the formation of the alkylated products and also the oxygenated products (histogram in Fig. 9B). At temperature of $300^{\circ}$, OMS-2RS sample shows the highest selectivity towards the formation of cyclohexene, while OMS-2RC shows the highest selectivity for oxygenated products formation, in agreement with the results obtained by Abdel Dayem et al. [31], who prepared Ni/alumina catalyst doped with different rare earth metals and tested for cyclohexane 
(a)

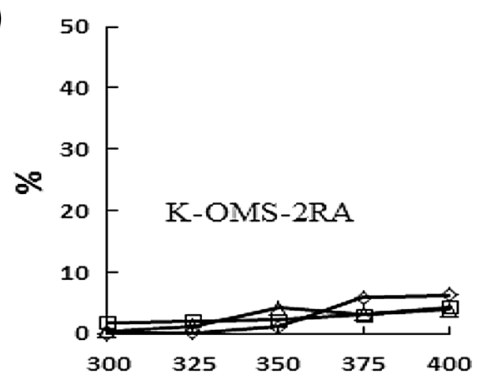

(b)

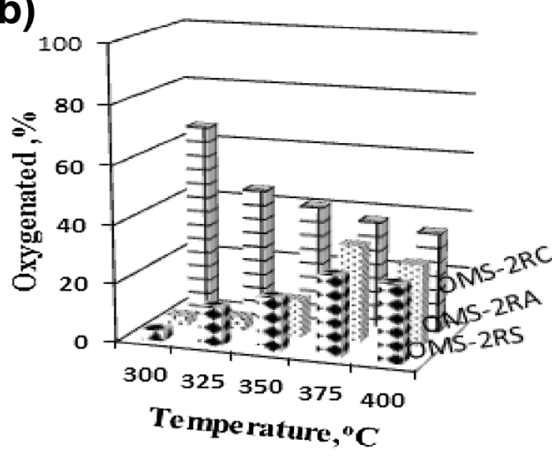

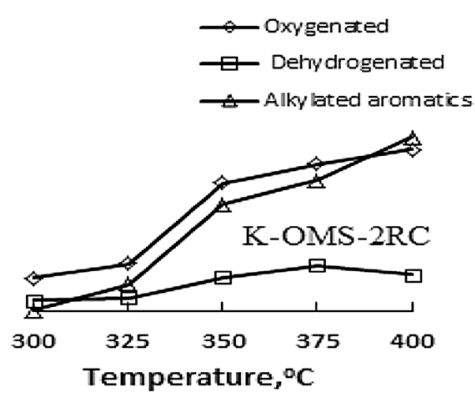

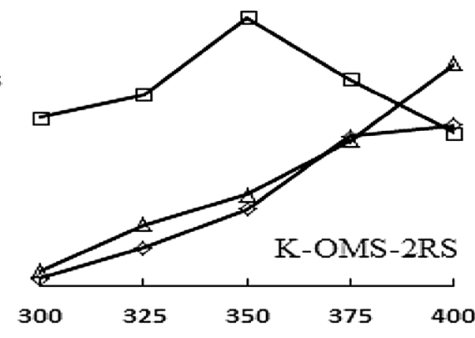

Temperature, ${ }^{\circ} \mathrm{C}$
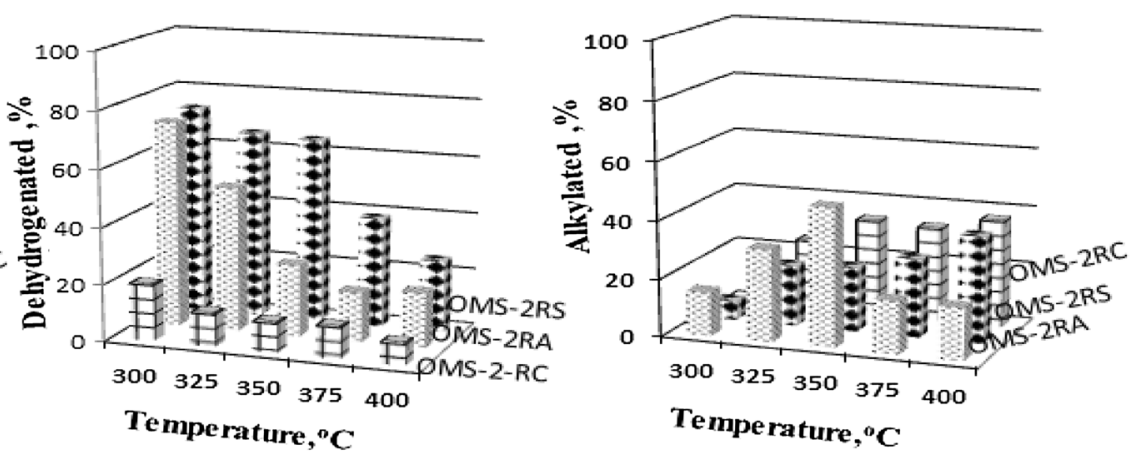

Fig. 9 Effect of reaction temperature on $\mathbf{a}$ the distribution and $\mathbf{b}$ the selectivity of the converted products over the K-OMS-2R samples

conversion, and Feng et al. [32], who prepared a series of manganese metalloporphyrins/ZnO and tested cyclohexane oxidation.

The high activity and selectivity of the OMS-2RS sample for cyclohexene formation at low and high temperature related to the high surface area allow the available active sites on the catalyst surface. Besides, high average pore diameter $\sim 12 \mathrm{~nm}$ allows the easy desorption of cyclohexene intermediate before completing the oxidation reaction to oxygenated products. The small average diameter for OMS-2RC allows long residence time of the reactant on the active sites of the sample to complete its oxidation cycle to oxygenated products, besides the feasibility of lattice oxygen (as established from TPR results) which facilitates their contribution to the oxidation reaction and shows high selectivity and activity at low temperature.

\section{Reaction mechanism}

The mechanism proceeded as follows, in which adsorbed cyclohexane reacts with lattice oxygen and the reduced metal oxide reacts with adsorbed, dissociated oxygen. The oxidative dehydrogenation reaction mechanism is discussed as follows: gaseous oxygen participates in the reaction only after adsorption in other parts of the catalyst and then migrates through the lattice to the active sites by the re-oxidation between $\mathrm{Mn}^{3+}$ and $\mathrm{Mn}^{4+} \cdot \mathrm{Mn}^{4+}-\mathrm{O}$ is responsible for providing the centre of oxidation for the adsorbed alkyl species, which contributes to a high conversion of cyclohexane, whereas $\mathrm{Mn}^{3+}-\mathrm{O}$ is responsible for activating cyclohexane and adsorbing oxygen, which attributes a high selectivity to cyclohexene (oxidative dehydrogenated product).

Besides, the facility of oxygen dissociation occurred in metal-based catalyst by transferring an electron to metal centre, which is then back donated to the anti-bonding orbital of the molecule oxygen molecule. Cyclohexene is oxidized to cyclohexanol by the assistance of lattice oxygen and then dehydrogenated to cyclohexanon (oxygenated products).

\section{Conclusion}

Needle-like nanorods of cryptomelane-type manganese oxide K-OMS-2 nanomaterials were prepared via reflux of $\mathrm{Mn}^{+2}$ and $\mathrm{Mn}^{+7}$ in acidic medium using different manganese anions precursors (sulphate, chloride and acetate).

From the previous results, it was clarified that the nature of the anions played an important role in the structural characterization and the catalytic activity of the prepared cryptomelane sample as emphasized by the following.

The three different manganese anion precursors used for the preparation of the cryptomelanes produce a similar crystalline phase with minor differences in crystallite size and minor morphological variations coupled with different 
nitrogen adsorptions in the three cryptomelane structures. The sample prepared using manganese acetate precursor contains the highest tunnel hydroxyl group and is a thermally stable one. The cryptomelane sample prepared using manganese sulphate as a precursor has more number of lattice oxygen species. In addition, the K-OMS-2RS sample exhibits high BET surface area, total pore volume and average pore diameter compared with the other two samples.

Although, K-OMS-2RA has the highest reducibility (as shown in TPR), it shows the lowest catalytic activity. The oxidation reaction of the cyclohexane is considered as a structure-sensitive reaction. The large pore diameter could be a reason why K-OMS-2RS was high in cyclohexane conversion. Besides, the loss of this oxygen creates nucleophilic vacancies in the lattice, implying their participation in the oxidation reaction of the cyclohexane.

Open Access This article is distributed under the terms of the Creative Commons Attribution 4.0 International License (http://crea tivecommons.org/licenses/by/4.0/), which permits unrestricted use, distribution, and reproduction in any medium, provided you give appropriate credit to the original author(s) and the source, provide a link to the Creative Commons license, and indicate if changes were made.

\section{References}

1. Hattori, H., Ide, Y., Ogo, S., Inumaru, K., Sadakane, M., Sano, T.: Efficient and selective photocatalytic cyclohexane oxidation on a layered titanate modified with iron oxide under sunlight and $\mathrm{CO}_{2}$ atmosphere. ACS Catal. 2, 1910-1915 (2012)

2. Modi C, Trivedi P.: Zeolite-Y entrapped Ru(III) and Fe(III) complexes as heterogeneous catalysts for catalytic oxidation of cyclohexane reaction. Arab. J. Chem. 2013 (Under press)

3. Suib, S.: Porous manganese oxide octahedral molecular sieves and octahedral layered materials. Acc. Chem. Res. 41, 479-487 (2008)

4. Peluso, M., Gambaro, L., Pronsato, E., Gazzoli, D., Thomas, H., Sambeth, J.: Synthesis and catalytic activity of manganese dioxide (type OMS-2) for the abatement of oxygenated VOCs. Catal. Today 133, 487-492 (2008)

5. Dharmarathna, S., Kingóndu, C., Pahalagedara, L., Kuo, C., Zhang, Y., Suib, S.: Manganese octahedral molecular sieve (OMS-2) catalysts for selective aerobic oxidation of thiols to disulfides. Appl. Catal. B: Environ. 147, 124-131 (2014)

6. Fan, C., Lu, A., Li, Y., Wang, C.: Synthesis, characterization, and catalytic activity of cryptomelane nanomaterials produced with industrial manganese sulphate. J. Colloid Interf. Sci. 327, 393-402 (2008)

7. Tian, H., He, J., Zhang, X., Zhou, L., Wang, D.: Facile synthesis of porous manganese oxide K-OMS-2 materials and their catalytic activity for formaldehyde oxidation. Microporous Mesoporous Mater. 138, 118-122 (2011)

8. Sun, H., Chen, S., Wang, P., Quan, X.: Catalytic oxidation of toluene over manganese oxide octahedral molecular sieves (OMS-2) synthesized by different methods. Chem. Eng. J. 178, 191-196 (2011)

9. Schurz, F., Bauchert, J., Thorsten, M., Schleid, T., Hasse, H., Gläser, R.: Octahedral molecular sieves of the type K-OMS-2 with different particle sizes and morphologies: impact on the catalytic properties in the aerobic partial oxidation of benzyl alcohol. Appl. Catal. A: Gen. 355, 42-49 (2009)

10. Sithambarama, S., Wenc, W., Njagia, E., Shenb, X., Hansonc, J., Suiba, $\mathrm{S} .: \mathrm{H}_{2}$ production through the water-gas shift reaction: an in situ time-resolved X-ray diffraction investigation of manganese OMS-2 catalyst. Catal. Today 156, 2-7 (2010)

11. Sun, M., Yu, L., Ye, F., Diao, G., Yu, Q., Hao, Z., Zheng, Y., Yuan, L.: Transition metal doped cryptomelane-type manganese oxide for low-temperature catalytic combustion of dimethyl ether. Chem. Eng. J. 220, 320-327 (2013)

12. Deng, Y., Zhang, T., Au, C., Yin, S.: Oxidation of p-chlorotoluene to $\mathrm{p}$-chlorobenzaldehyde over manganese-based octahedral molecular sieves of different morphologies. Catal. Commun. 43, 126-130 (2014)

13. Hndel, M., Rennert, T., Totsche, K.: Synthesis of cryptomelaneand birnessite-type manganese oxides at ambient pressure and temperature. J. Colloid Interf. Sci. 405, 44-50 (2013)

14. Almquist, C., Krekeler, M., Jiang, L.: An investigation on the structure and catalytic activity of cryptomelane-type manganese oxide materials prepared by different synthesis routes. Chem. Eng. J. 252, 249-262 (2014)

15. Duan, L., Sun, B., Wei, M., Luo, S., Pan, F., Xu, A., Li, X.: Catalytic degradation of Acid Orange 7 by manganese oxide octahedral molecular sieves with peroxymonosulfate under visible light irradiation. J. Hazard. Mater. 285, 356-365 (2015)

16. DeGuzman, R., Shen, Y., Neth, E., Suib, S., O’Young, C., Levine, S., Newsam, J.: Synthesis and characterization of octahedral molecular sieves (OMS-2) having the hollandite structure. Chem. Mater. 6, 815-821 (1994)

17. Gac, W.: The influence of silver on the structural, redox and catalytic properties of the cryptomelane-type manganese oxides in the low-temperature $\mathrm{CO}$ oxidation reaction. Appl. Catal. B: Environ. 75, 107-117 (2007)

18. Julien, C., Massot, M., Poinsignon, C.: Profil enregistré d'une raie spectrale photographique et "largeur effective" Spectrochim. Acta A 60, 689-694 (2004)

19. Hndel, M., Rennert, T., Totsche, K.: Synthesis of cryptomelaneand birnessite-type manganese oxides at ambient pressure and temperature. J. Coll. Interf. Sci. 405, 44-50 (2013)

20. Chen, C., Njagi, Chen, S., Horvath, D., Morey, A., Mackin, C., Joesten, R., Suib, S.: Structural distortion of molybdenum-doped manganese oxide octahedral molecular sieves for enhanced catalytic performance. Inorg. Chem. 54, 10163-10171 (2015)

21. Julien, C., Massot, M., Poinsignon, C.: Lattice vibrations of manganese oxides: part I. Periodic structures. Spectrochim. Acta A 60, 689-700 (2004)

22. Lan, W., Chen, C.: Hybridization of graphene in 3D complex nanovoids: synergistic nanocomposites for electrocatalytic reduction of hydrogen peroxide. Electrochim. Acta 180, 1014-1022 (2015)

23. Gao, T., Glerup, M., Krumeich, F., Nesper, R., Fjellvg, H., Norby, P.: Microstructures and spectroscopic properties of cryptomelane-type manganese dioxide nanofibers. J. Phys. Chem. C 112, 13134-13140 (2008)

24. Cheng, F., He, C., Shu, D., Chen, H., Zhang, J., Tang, S.: Preparation of nanocrystalline $\mathrm{VN}$ by the melamine reduction of $\mathrm{V}_{2} \mathrm{O}_{5}$ xerogel and its supercapacitive. Mater. Chem. Phys. 131, 268-273 (2011)

25. Walanda, D., Lawrance, G., Donne, S.: Hydrothermal $\mathrm{MnO}_{2}$ : synthesis, structure, morphology and discharge performance. J. Power Source 139, 325-341 (2005)

26. Liu, X., Jin, Z., Lu, J., Wang, X., Luo, M.: Highly active CuO/ OMS-2 catalysts for low-temperature CO oxidation. Chem. Eng. J. 162, 151-157 (2010) 
27. Kuo, C., Lan, W., Chen, C.: Redox preparation of mixed-valence cobalt manganese oxide nanostructured materials: highly efficient noble metal-free electrocatalysts for sensing hydrogen peroxide. Nanoscale 6, 334-341 (2014)

28. Brunauer, S., Deming, S., Deming, E., Teller, E.: On a theory of the van der waals adsorption of gases. J. Am. Chem. Soc. 62, 1723-1728 (1940)

29. Malinger, K., Ding, Y., Sithambaram, S., Espinal, L., Gomez, S., Suib, S.: Microwave frequency effects on synthesis of cryptomelane-type manganese oxide and catalytic activity of cryptomelane precursor. J. Catal. 239, 290-298 (2006)
30. Gregg, S., Sing, K.: Adsorption surface area and porosity, 2nd edn. Academic Press Inc., London (1982)

31. Abdel Dayem, H., Faiz, M., Abdel-Samad, H., Hassan, S.: Rare earth oxides doped $\mathrm{NiO} / \gamma-\mathrm{Al}_{2} \mathrm{O}_{3}$ catalyst for oxidative dehydrogenation of cyclohexane. J. Rare Earths 33, 611-617 (2015)

32. Feng, Z., Xie, Y., Hao, F., Liu, P., Luo, H.: Catalytic oxidation of cyclohexane to KA oil by zinc oxide supportedmanganese 5, 10, 15, 20-tetrakis (4-nitrophenyl)porphyrin. J. Mol. Catal. A: Chem. 410, 221-225 (2015) 